\title{
Right Salpingectomy
}

National Cancer Institute

\section{Source}

National Cancer Institute. Right Salpingectomy. NCI Thesaurus. Code C51606.

Surgery to remove part or all of the right fallopian tube. 